\title{
Capacidade resistente e modos de ruptura de vigas de concreto armado reforçadas à flexão com fibras de carbono
}

\section{Resistant capacity and failure modes of reinforced concrete beams strengthened in flexure with externally bonded carbon fiber reinforced plastic}

\begin{abstract}
Augusto Ottoni Bueno da Silva
Doutorando do Departamento de Estruturas da Faculdade de Engenharia Civil e Arquitetura da Universidade Estadual de Campinas - Unicamp. augusto.ottoni@terra.com.br
\end{abstract}

\footnotetext{
Armando Lopes Moreno Júnior

Pós-doutor em Engenharia Civil, Professor da

Faculdade de Engenharia Civil e Arquitetura da Universidade Estadual de Campinas - Unicamp. almoreno@fec.unicamp.br
}

Gisleiva Cristina dos Santos Ferreira

Doutora em Engenharia Civil,

Professora da Faculdade de Tecnologia da

Universidade Estadual de Campinas - Unicamp. gisleiva@ft.unicamp.br

\section{Resumo}

Para se projetar um reforço à flexão com material polimérico reforçado com fibras, em uma viga de concreto armado, é necessário que o engenheiro responsável opte por trabalhar sem a utilização de mecanismos de incremento de ancoragem, obviamente dentro de níveis seguros, ou utilize artifícios que lhe garantam alcançar a deformação última de projeto da fibra fornecida pelo fabricante da manta. Nesse artigo, é apresentada a base para o cálculo das duas metodologias, segundo os critérios mais expressivos existentes, e são feitas comparações com resultados experimentais. Para o reforço sem ancoragem, verificou-se que, para se evitarem as indesejadas rupturas prematuras e bruscas, as metodologias propostas pelos ACI e FIB mostraram-se adequadas. Para se garantir a deformação última da fibra, foi estudado um mecanismo com ancoragem lateral a $45^{\circ}$ feito com o próprio material da fibra. Nesse caso, utilizou-se o processo de seções balanceadas, para a previsão do modo de ruptura, e, em seguida, foram feitas as devidas comparações entre os valores esperados com os resultados obtidos nos ensaios em laboratório.

Palavras-chave: Viga de concreto armado, fibra de carbono, reforço estrutural.

\begin{abstract}
To design an externally bonded fiber reinforced plastic reinforcement for a reinforced concrete beam, it is necessary that the engineer responsible chooses to work without the use of mechanisms to increase the anchoring within safe levels, or make use of artifacts to ensure that the deformation in the fiber reaches ultimate state value supplied by the manufacturer. This article shows the basis for the calculation of the two methodologies under the existing criteria more expressively and also makes comparisons with laboratory tests. For enhancement without anchoring, it was found that to prevent unwanted premature and abrupt ruptures, the methods proposed by ACI and FIB showed to be adequate. To ensure the ultimate deformation of the fiber, a mechanism for anchoring in the form of a bow $45^{\circ}$ made by the material of the fiber was studied. In this case, the process of balanced sections to foresee the mode of collapse were verified and then the appropriate comparisons were made between the expected values with the tests results found in the laboratory.
\end{abstract}

Keywords: Reinforced concrete beam, carbon fiber, structural reinforcement. 


\section{Introdução}

Entre os atuais usos do Polímero Reforçado com Fibras de Carbono (PRFC), pode-se citar as vigas armadas com barras poliméricas (Ashour \& Family, 2006), as vigas reforçadas com multi-armaduras aço-PRFC (Si-Larbi et al., 2006) e o reforço de superfície de alvenarias estruturais não armadas $(\mathrm{Pa}-$ panicolaou et al., 2008). Como principal alternativa à colagem de PRFC, na superfície de peças estruturais de concreto armado, tem-se estudado a sua aplicação na camada de cobrimento de vigas (near surface mounted) (Barros \& Dias, 2006). Essa técnica consiste em executar sulcos na região do cobrimento sobre o qual são posicionadas e coladas tiras de reforço. Apesar desses grandes avanços, pesquisadores estudam, ainda, como prever a capacidade última e como calcular os modos de ruptura de vigas reforçadas externamente à flexão com PRFC.

Vários pesquisadores já verificaram a ocorrência de rupturas prematuras relacionadas ao descolamento abrupto da camada de reforço ou mesmo ao arrancamento do cobrimento do concreto no nível da armadura (Saadatmanesh \& Ehsani, 1991). Por esses motivos, estudos já foram realizados para estabelecer mecanismos de ancoragem que evitem o descolamento prematuro da manta, de maneira a possibilitar que o reforço trabalhe com sua plena capacidade (Arduini et al., 1997; Norris et al., 1997), porém os métodos apresentados não são acompanhados de uma marcha de cálculo explicando como foram dimensionados esses mecanismos.

Nesse trabalho, é proposto um mecanismo de ancoragem lateral, feito com a própria manta de PRFC, colado a $45^{\circ}$ em relação à face inferior da viga. A metodologia adotada para o dimensionamento do laço tem como objetivos minimizar os gastos com material, fazer com que a manta não se descole abrupta- mente e possibilitar que a peça alcance a sua capacidade resistente de projeto.

Além disso, é preciso garantir uma ruptura dúctil para a peça reforçada no estado-limite último. Esse aspecto foi estudado a partir de uma proposta dos pesquisadores Ng e Lee (2002), sendo os valores esperados comparados com os resultados experimentais do ensaio de três vigas reforçadas.

Esse trabalho teve como intuito, ainda, estudar o descolamento abrupto da manta, quando não existe esse mecanismo de ancoragem em laço. Para isso, os resultados experimentais de uma viga reforçada sem laço foram comparados com as prescrições das normas ACI (ACI, 1996) e FIB (FIB, 2001) e com a marcha de cálculo proposta por Chaallal e equipe (Chaallal et al., 1998).

Para efeito de comparação dos estudos propostos com resultados experimentais, foram suprimidos os coeficientes de segurança típicos de projeto.

\section{Previsão da capacidade resistente de vigas reforçadas externamente à flexão com a utilização de mecanismos de ancoragem}

\section{Previsão do modo de ruptura}

Com a utilização de mecanismos de ancoragem, espera-se que a manta alcance a sua deformação última. Supondo, então, que a manta não irá descolar, os pesquisadores Ng e Lee (2002) sugerem que sejam previstas áreas de seção transversal de fibra (máximas e mínimas), para se assegurar que as vigas reforçadas irão romper de uma maneira dúctil. Assim, são definidas as seções balanceadas e as regiões de projeto (Figura 1). Também são definidas as deformações na seção transversal da viga reforçada (Figura 2).

A seção balanceada 1 define a transição entre as regiões 1 e 2 de projeto. Nessa seção, $\varepsilon_{\mathrm{c}}=\varepsilon_{\mathrm{cu}}, \varepsilon_{\mathrm{s}}>\varepsilon_{\mathrm{y}}, \varepsilon_{\mathrm{s}}=\varepsilon_{\mathrm{y}}, \mathrm{e}$, $\varepsilon_{\mathrm{f}}<\varepsilon_{\mathrm{fu}}$. A seção balanceada 2 define a transição entre as regiões 2 e 3 . Nessa seção, $\varepsilon_{\mathrm{c}}=\varepsilon_{\mathrm{cu}}, \varepsilon_{\mathrm{s}}>\varepsilon_{\mathrm{y}}, \varepsilon_{\mathrm{s}}>\varepsilon_{\mathrm{y}}, \mathrm{e}, \varepsilon_{\mathrm{f}}=\varepsilon_{\mathrm{fu}} \cdot \mathrm{Na}$ região 1 , ocorre a ruína por ruptura do concreto, sendo que a armadura inferior não terá, ainda, entrado em regime de escoamento. $\mathrm{Na}$ região 2 , a ruína ocorre pelo escoamento da armadura longitudinal inferior, sem a manta ter alcançado sua deformação última. Por fim, na região 3, por deficiência de área de fibra e pelo subaproveitamento do concreto, a ruína se dá por ruptura

brusca da manta. Para efeito de projeto, o ideal é que se trabalhe, na região 2, mais especificamente na transição entre as regiões 2 e 3, o que leva a se calcular a peça balizando-se na seção balanceada 2 .

Para se montar o diagrama ilustrado na Figura 1, deve-se determinar as posições da linha neutra para a seção balanceada $1\left(\mathrm{x}_{\mathrm{sb} 1}\right)$ e $2\left(\mathrm{x}_{\mathrm{sb} 2}\right)$. Esse cálculo é feito utilizando-se as Equações $1 \mathrm{e} 2$. Em seguida, com a equação de equilíbrio de forças da seção, iguala-se a área de aço a zero $\left(A_{s}=0\right)$ e encontra-se a área de fibra, $\mathrm{A}_{\mathrm{f}}$ (pontos A e C no diagrama), e,

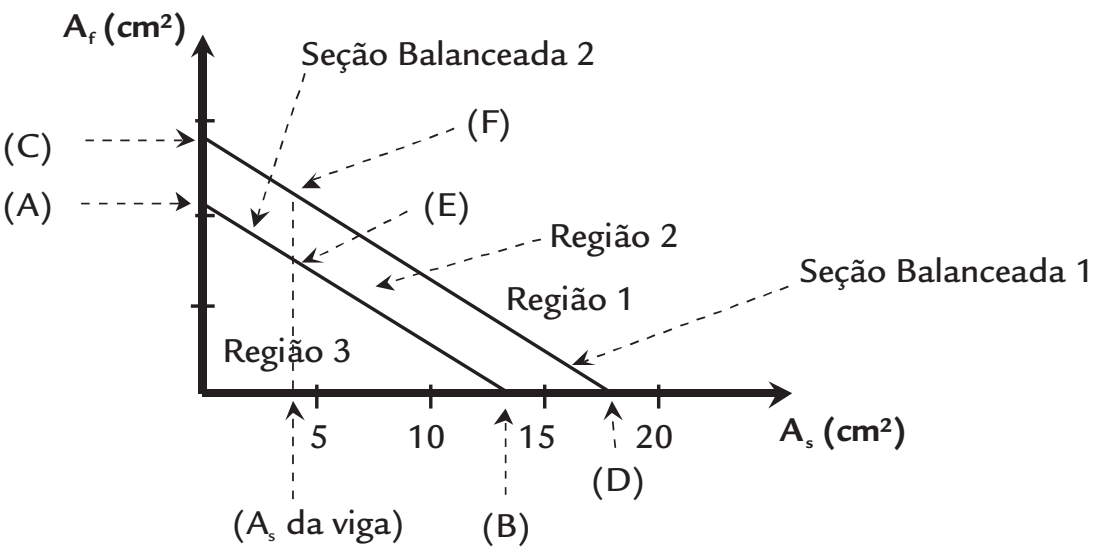

Figura 1

Modos de ruptura e seções balanceadas para vigas reforçadas com PRFC. 
Figura 2

Deformações na seção transversal de viga reforçada com PRFC.

em seguida, faz-se $A_{f}=0$ e encontra-se $A_{s}$ (pontos B e D). Com o valor da área da

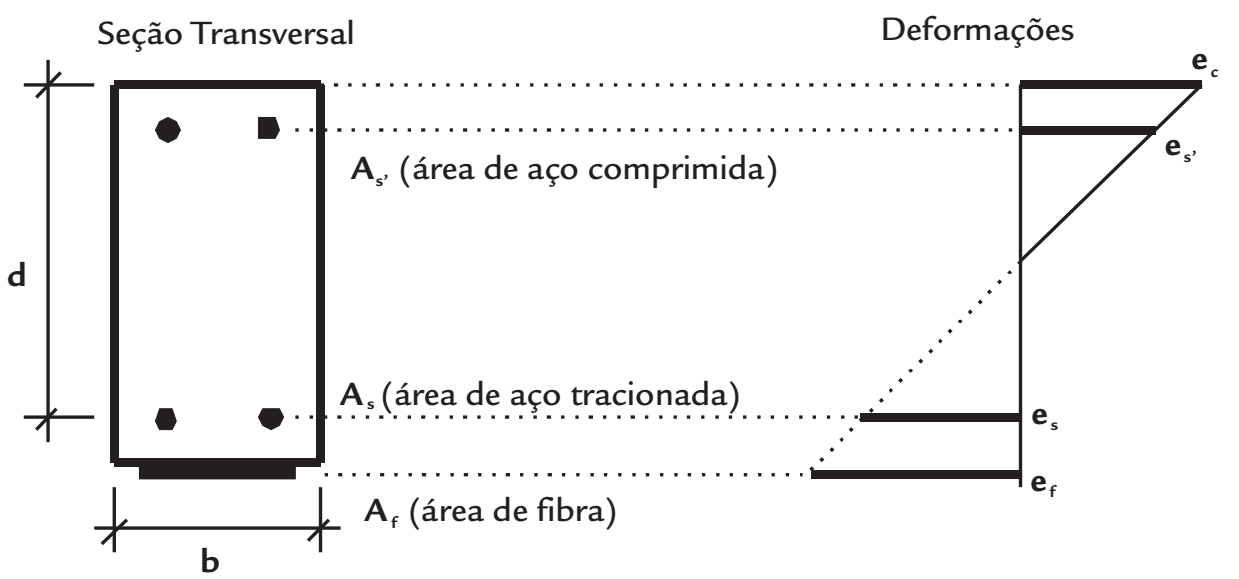

Deformações-limites:

$\mathbf{e}_{\mathrm{cu}}=$ deformação última do concreto.

$\mathbf{e}_{y^{\prime}}=$ deformação de escoamento da armadura comprimida.

$\mathbf{e}_{\mathrm{y}}=$ deformação de escoamento da armadura tracionada.

$\mathbf{e}_{\mathrm{fu}}=$ deformação última da fibra.

armadura longitudinal inferior da viga que irá ser reforçada, determinamos os

$$
\begin{gathered}
\xi_{1}=\frac{x_{s b 1}}{d}=\frac{\varepsilon_{c u}}{\varepsilon_{c u}+\varepsilon_{y}} \\
\xi_{2}=\frac{x_{s b 2}}{d}=\frac{\varepsilon_{c u}}{\varepsilon_{c u}+\varepsilon_{f}} \frac{A_{f}}{b . d}
\end{gathered}
$$

pontos E e F, delimitadores da região de projeto 2 .

\section{O Mecanismo de ancoragem}

A partir da análise das experiências obtidas por vários pesquisadores, é proposta, aqui, a utilização da própria manta como um mecanismo de reforço, sendo apresentado a seguir a sua metodologia de cálculo. O dimensionamento dos dispositivos laterais, que funcionam como incremento de ancoragem, pode ser baseado em duas suposições, confor-

me descrito em Silva (2001). Na primeira, supõe-se que a manta não descola da parte inferior da viga, o que faz com que o valor da força $T_{f}$, que balizará o cálculo, deva ser tomado no ponto $\mathrm{B}$, indicado na Figura 3A. Essa situação será denominada de Primeiro Caso. A segunda suposição baseia-se no fato de que a manta se descola por completo da face inferior da

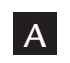

A

Figura 3

Suposições para o dimensionamento das ancoragens laterais.

A) Primeiro caso.

B) Segundo caso.

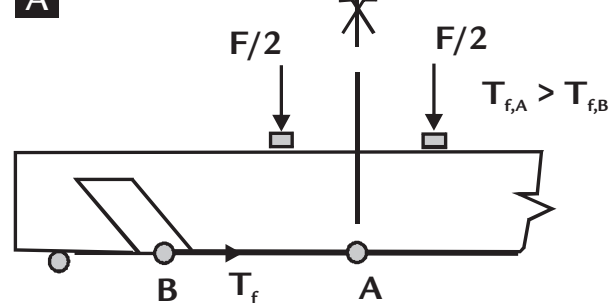

viga, na região entre as ancoragens do lado esquerdo e do lado direito da peça, o que faz com que a manta trabalhe como um tirante, que está sendo seguro justamente pelas ancoragens. Nessa situação, aqui denominada de Segundo Caso, a força $T_{f}$, que age na ancoragem, é a mesma que está atuando no meio do vão, no ponto A, como ilustrado na Figura 3B.

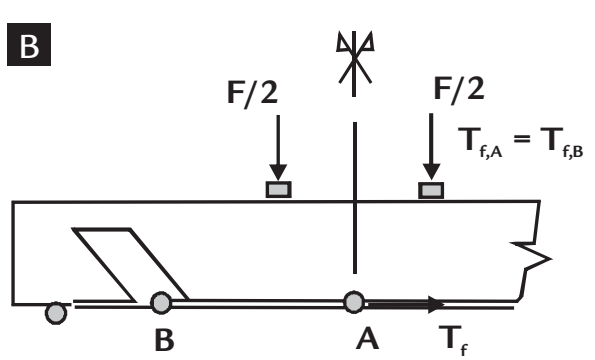

\section{Dimensionamento da ancoragem lateral: primeiro caso}

O primeiro passo para o dimensionamento é determinar a posição, em relação ao apoio, em que a força no polímero reforçado com fibras, $\mathrm{T}_{\mathrm{f}}$, está atuando. Para isso, precisa-se, a princípio, supor uma determinada largura para a ancoragem, para se determinar a posição na qual se calcula $\mathrm{T}_{\mathrm{f}}$. Além disso, deter- minou-se que o ângulo de inclinação da ancoragem, em relação ao eixo longitudinal da viga, seria $45^{\circ}$, conforme Figura 4. O segundo passo consta da obtenção de $T_{f}$. Para isso, será utilizada a equação de equilíbrio de momentos (Equação 3), para a determinação da posição da linha neutra, e, em seguida, monta-se um siste- ma de duas equações, compatibilidade de deformações (Equação 4) e equilíbrio de forças (Equação 5), a duas incógnitas, $\varepsilon_{\mathrm{s}} \mathrm{e}$ $\varepsilon_{\mathrm{f}}$. Encontrado o valor de $\varepsilon_{\mathrm{f}}$, determina-se a tensão na fibra, $\sigma_{\mathrm{f}}$, através da Equação 6. E, finalmente, com o valor de $\sigma_{f}$, determina-se $T_{f}$ atuante na posição $B$, através da Equação 7. 


$$
\begin{gathered}
M=b_{w} \cdot 0,8 \cdot x \cdot f_{c} \cdot\left[\left(h+\frac{t_{f}}{2}\right)-\frac{0,8 \cdot x}{2}\right]-A_{s} \cdot \sigma_{s} \cdot\left[(h-d)+\frac{t_{f}}{2}\right] \\
\frac{\varepsilon_{s}}{d-x}=\frac{\varepsilon_{f}}{\left(h+\frac{t_{f}}{2}\right)-x} \\
0=b_{w} \cdot 0,8 \cdot x \cdot f_{c}-A_{s} \cdot \sigma_{s}-A_{f} \cdot \sigma_{f} \\
\sigma_{f}=E_{f} \cdot \varepsilon_{f} \\
T_{f}=\sigma_{f} \cdot A_{f}
\end{gathered}
$$

Com o valor de $T_{f}$ obtido, os dois últimos passos são as verificações da ruptura e do cisalhamento. É durante a verificação da ruptura, que é feita primeiro,

Encontrando-se o valor da largura da ancoragem, faz-se a verificação ao descolamento, que é o último passo a ser dado. A verificação é feita comparando- que se supõe um valor para a largura da ancoragem. Para esse cálculo, rebate-se metade do valor da força $T_{f}$ (Figura 4) na direção de $R_{1, \text { ancoragem }}$, que é a força

$$
R_{1, \text { ancoragem }} \geq \frac{T_{f}}{2} \cdot \cos 45^{\circ}
$$

se a força resistente ao cisalhamento com a força atuante. As forças resistente e atuante são obtidas através das Equações 9 e 10, respectivamente. Para o cálculo da

$$
\begin{gathered}
F_{\text {resistente }}=\tau_{m} \cdot A_{1, \text { ancoragem }} \\
F_{\text {atuante }}=\frac{T_{f}}{2} \cdot \cos 45^{\circ}
\end{gathered}
$$
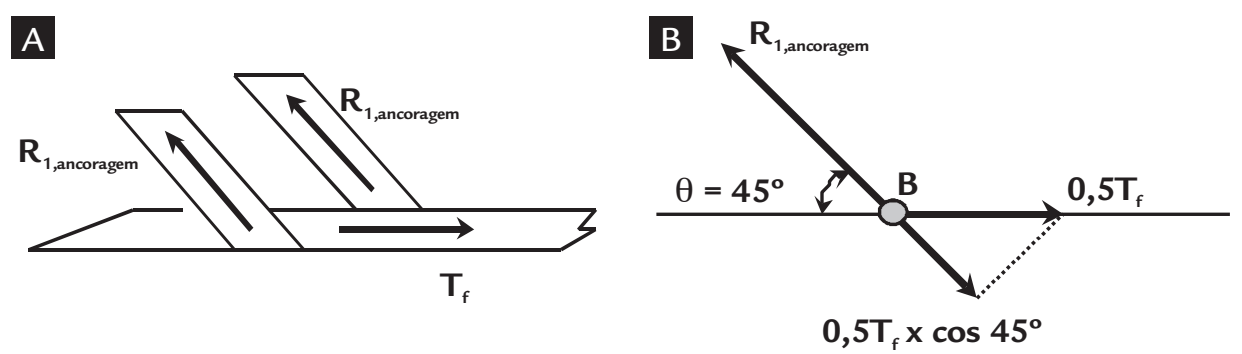

resistida por uma ancoragem, e faz-se a verificação segundo a Equação 8 . força resistente, foi adotada uma tensão média resistente ao cisalhamento, $\tau_{m}$, dividindo por dois o valor de $\tau_{i, d e s c}$.
Figura 4

Verificação da ruptura e do cisalhamento: a força resistente em cada ancoragem, $R_{1, \text { ancoragem }}$, comparada com metade da força atuante na manta.

\section{Dimensionamento da ancoragem lateral: segundo caso}

Desprezando o fato da curvatura apresentada pela viga fletida, a manta está trabalhando como um tirante, sendo que a força atuante na posição $\mathrm{A}$ pode ser considerada como a mesma que está atuando na posição $\mathrm{B}$, que é o local onde se deve calcular $T_{f}$ (Figura 3B). Primeiro determina-se o valor de $T_{f}$, na posição A. Em seguida, faz-se a verificação da ruptura e, finalmente, a verificação do descolamento.

Com o valor de $\varepsilon_{\mathrm{fu}}$, determina-se a tensão na manta. Multiplicando-se pela área de fibra, $A_{f}$, tem-se $T_{f}$ Determina-se

\section{Previsão da capacidade resistente de vigas reforçadas externamente à flexão sem a utilização de mecanismos de ancoragem}

A previsão da capacidade resistente de vigas sem mecanismos de ancoragem será feita de acordo com as marchas de cálculo propostas pelo ACI (ACI, 1996), pela Féde-

\section{Programa experimental}

O programa experimental constou da análise de cinco vigas retangulares de concreto armado de alta resistência, com mesma geometria e armaduras, longitu- a largura da ancoragem lateral, utilizando-se, também, a Inequação 08. Com a largura calculada, faz-se a verificação do descolamento, novamente comparando o valor da força resistente com o valor da força atuante.

ration Internationale du Béton (FIB, 2001) e por Chaallal e equipe (Chaallal et al., 1998). dinal ( 2 barras de $8 \mathrm{~mm}$, CA50) e transversal (barras de 6,3 $\mathrm{mm}$ a cada $10 \mathrm{~cm}$, 
CA60), sendo quatro delas reforçadas à flexão com mantas de PRFC.

Das vigas analisadas experimentalmente, uma era testemunho, e foi designada como viga VT; uma foi reforçada por meio da colagem de uma camada de manta de PRFC na face inferior da peça, VR1C; outras três vigas, além da manta de PRFC na face inferior da peça, receberam, ainda, mecanismos de incremento de ancoragem lateral, também com manta de PRFC, na forma de um "X", nas extremidades de cada viga, próximas às regiões dos apoios, sendo denominadas VR1C/X (Figura 5), VR2C/X e VR4C/X, vigas estas reforçadas com uma, duas e quatro camadas de mantas, respectivamente.

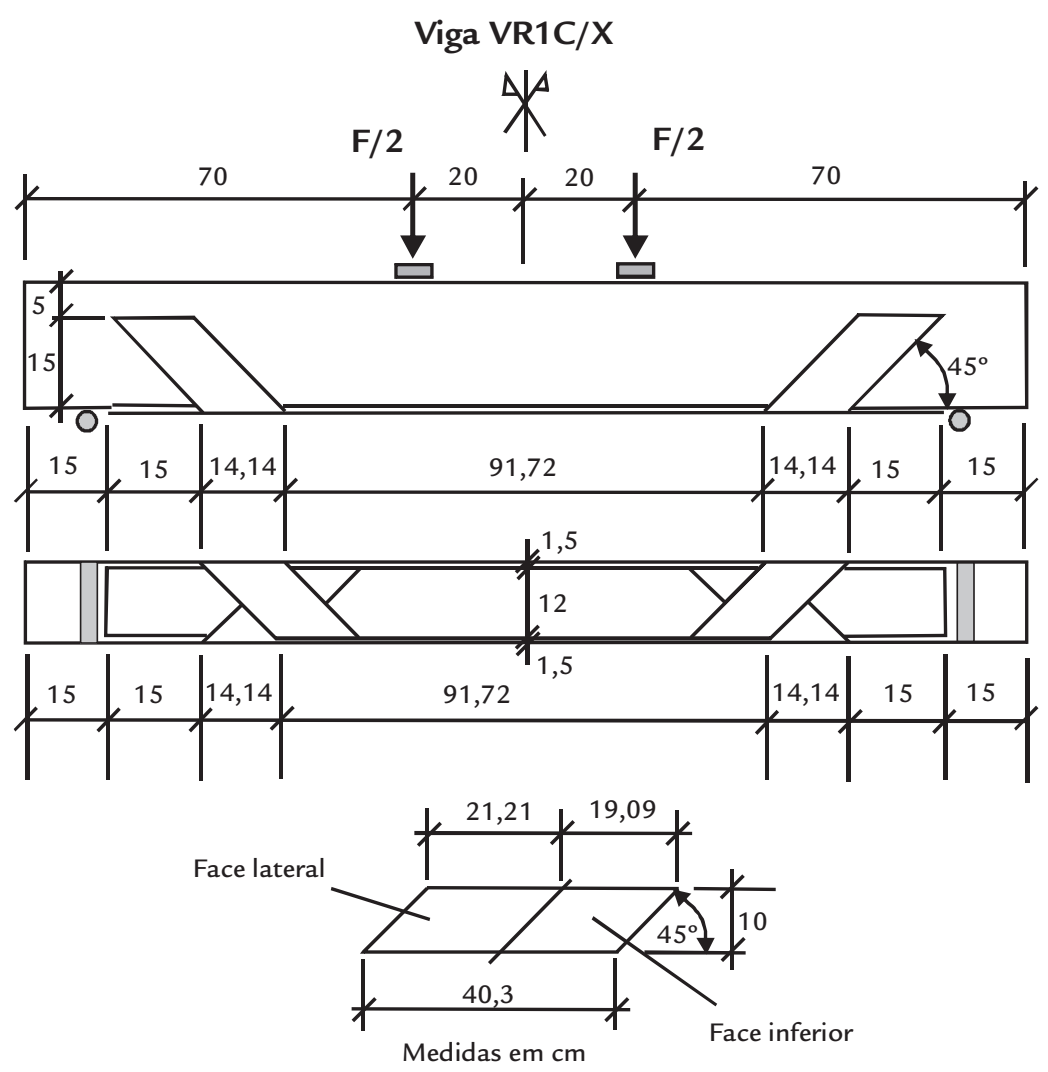

Foram realizados ensaios com corpos de prova para a caracterização dos materiais. Para a armadura longitudinal foram obtidos: $f_{y}=514,5 \mathrm{MPa}, e_{\gamma}=0,25 \%$ e $E_{s}=205,8 \mathrm{GPa}$; para a armadura transversal, $f_{s w}=660 \mathrm{MPa}, e_{s w}=0,48 \%$ e $E_{s w}=229,65 \mathrm{GPa}$ e, para o concreto, $f_{c, 28}=90,3 \mathrm{MPa}$ e $E_{c, 28}=39,3 \mathrm{GPa}$. No caso da manta, o fabricante sugere, para previsão do momento-fletor último, que se trabalhe com uma deformação de

$70 \%$ da deformação de ruptura obtida em ensaio à tração simples. Como foi registrada deformação máxima de $0,81 \%$, adotou-se, para a deformação última $\left(e_{f u}\right)$, o valor de $0,57 \%$.

Os valores calculados e utilizados para as larguras das ancoragens laterais, de acordo com a metodologia apresentada, estão especificados na Tabela 1. Foram utilizados os valores de módulos de elasticidade e de cisalhamento do adesivo, respectivamente, $E=724,8$ $\mathrm{N} / \mathrm{mm}^{2}$ e $G=278,77 \mathrm{~N} / \mathrm{mm}^{2}$, momento de inércia da manta $I_{\text {manta }}=0,016$ $\mathrm{mm}^{4}$, espessura de cálculo da manta $=0,117 \mathrm{~mm}$ e espessura física, ou seja, pós-impregnação $=0,4 \mathrm{~mm}$. Para não ocorrer rompimento ou descolamento das ancoragens e garantir que a manta alcançasse sua deformação última, foi adotado um fator de segurança 2 , para a largura das ancoragens.

Tabela 1

Valores de $b_{1, \text { ancoragem }}$ necessários segundo as duas metodologias propostas e os valores efetivamente escolhidos para serem utilizados.

\section{Resultados obtidos e discussão}

\section{Vigas com mecanismo de ancoragem}

Na Tabela 2, são apresentados os valores de momento-fletor esperados e obtidos nos ensaios para as três vigas com ancoragem, sendo $M_{i, f s}$ o momento-fletor de início de fissuração e $M_{y} \mathrm{o}$ momento-fletor de início de escoamento da armadura longitudinal inferior. Construindo-se o gráfico sugerido por $\mathrm{Ng}$ e Lee (2002), para as vigas reforçadas, chega-se ao apresentado na Figura 6.
Para as áreas de manta utilizadas, o diagrama de seções balanceadas previa, para os três ensaios vigas atingindo o estado-limite último na região 3 , conforme pontos G, H e I da Figura 6, com ruína da 
manta, sendo isto o que foi realmente observado nos ensaios. Como foi utilizado um concreto de elevada resistência e trabalhouse nos padrões de deformação da manta sugeridos pelo fabricante $\left(\varepsilon_{f}=0,57 \%\right)$, para o estado de deformação última, a viga não trabalhou na região de ruptura 2 .

Os valores de momento-fletor último esperados e obtidos em ensaio apresentaram erro máximo de $6 \%$, enquanto

\begin{tabular}{c|c|c|c|c|c}
\hline & Momentos (kN.m) & VT & VR1C/X & VR2C/X & VR4C/X \\
\hline \multirow{2}{*}{$\begin{array}{c}\text { Valores } \\
\text { esperados }\end{array}$} & $\mathrm{M}_{\text {u,esp }}$ & 8,85 & 13,30 & 17,73 & 26,50 \\
\cline { 2 - 6 } & $\mathrm{M}_{\text {máx,esp }}$ & - & 15,17 & 21,94 & 33,74 \\
\hline \multirow{4}{*}{ Ensaio } & $\mathrm{M}_{\mathrm{i}, \mathrm{fis}}$ & 5,08 & 5,80 & 6,53 & 7,25 \\
\cline { 2 - 6 } & $\mathrm{M}_{\mathrm{y}}$ & 7,32 & 10,50 & 11,50 & 14,79 \\
\cline { 2 - 6 } & $\mathrm{M}_{\mathrm{u}}$ & 9,83 & 13,94 & 16,60 & 25,29 \\
\cline { 2 - 6 } & $\mathrm{M}_{\text {máx }}$ & - & 16,07 & 24,65 & 34,80 \\
\hline
\end{tabular}

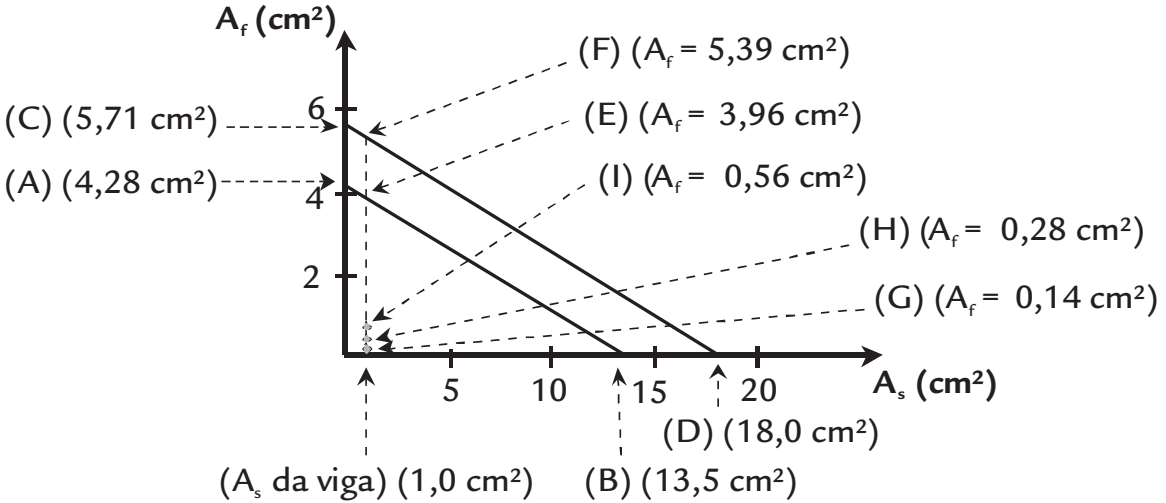

\section{Vigas sem mecanismo de ancoragem}

O momento-fletor de início de descolamento da manta observado no ensaio, $M_{i d}$, é determinado quando se verifica a disparidade entre as deformações $\mathrm{da}$ armadura longitudinal e da manta, denunciando o início do descolamento do material de reforço.

Comparando-se o valor encontrado, no ensaio da viga VR1C, com as metodologias do ACI, FIB e Chaallal, de acordo com a Tabela 3, percebe-se que os valores esperados pelo ACI e FIB, para o momento-fletor de início de descolamento, $M_{i d, e s p}$, mostraram-se seguros, pois, realmente, evitariam o descolamento, caso fossem adotados em projeto.

$\mathrm{O}$ valor do momento-fletor de início de descolamento sugerido por

\begin{tabular}{c|c|c|c}
\hline & Momentos (kN.m) & VT & VR1C \\
\hline \multirow{4}{*}{$\begin{array}{c}\text { Valores } \\
\text { esperados }\end{array}$} & $\mathrm{M}_{\mathrm{id}, \text { esp }}(\mathrm{ACl})$ & - & 10,24 \\
\cline { 2 - 4 } & $\mathrm{M}_{\mathrm{id} \text { esp }}(\mathrm{FIB})$ & - & 11,17 \\
\cline { 2 - 4 } & $\mathrm{M}_{\mathrm{id}, \text { esp }}($ Chaallal $)$ & - & 12,40 \\
\cline { 2 - 4 } & $\mathrm{M}_{\mathrm{u}, \text { esp }}$ & 8,85 & 13,30 \\
\cline { 2 - 4 } & $\mathrm{M}_{\text {máx,esp }}$ & - & 15,17 \\
\cline { 2 - 4 } & $\mathrm{M}_{\mathrm{id}}$ & - & 11,60 \\
\cline { 2 - 4 } & $\mathrm{M}_{\mathrm{u}}$ & 9,83 & não alcançado \\
\cline { 2 - 4 } & $\mathrm{M}_{\text {máx }}$ & - & não alcançado \\
\hline
\end{tabular}

\section{Conclusões}

Com relação às vigas com incremento de ancoragem, pode-se dizer que:

- A utilização de mecanismo de ancoragem lateral permite que seja alcançado o momento-fletor último, au- mentando a capacidade de carga das vigas. Conforme Tabela 2, a utilização de apenas uma camada de manta aumenta em $40 \%$ o momento-fletor último $(13,94 \mathrm{kN} . \mathrm{m} / 9,83 \mathrm{kN}$.m). Já a que, para os valores de momento-fletor máximo, a maior taxa de erro foi de $12 \%$ para a viga VR2C/X, provavelmente pelo descolamento da manta da face inferior da viga antes do esperado.

Tabela 2

Vigas VT, VR1C/X, VR2C/X e VR4C/X: valores dos momentos-fletores esperados e obtidos em ensaio.

Figura 6

Gráfico de Ng e Lee aplicado às vigas ensaiadas.

Chaallal $(12,4$ kN.m) apresentou-se cerca de $7 \%$ maior que o valor encontrado no ensaio, 11,60 kN.m $\left(M_{i d}\right)$, mostrando-se contra a segurança. Os valores de deformação última referente a $M_{u, e s p}$ e máxima, referente ao $M_{\text {máx,esp }}$, na manta, não foram alcançados, pois ocorreu o descolamento prematuro do reforço.

Tabela 3

Vigas VT e VR1C: valores dos momentosfletores esperados e obtidos em ensaio.

utilização de quatro camadas aumenta $257 \%$ o valor do momento fletor último $(25,29 \mathrm{kN} . \mathrm{m} / 9,83 \mathrm{kN} . \mathrm{m})$.

- Os valores de momento-fletor último esperados e obtidos em ensaios 
diferem em até $6 \%$, o que demonstra uma razoável concordância do modelo teórico utilizado com o que foi constatado durante os ensaios.

- A metodologia para a determinação das ancoragens laterais mostrou-se eficiente, sendo necessário que sejam feitos novos ensaios em que o intuito primordial seja o de calcular o limite de resistência das ancoragens, possibilitando verificar com precisão a marcha de cálculo proposta.
- A previsão do modo de ruptura proposta por $\mathrm{Ng}$ e Lee, quando não se corre o risco de haver rupturas prematuras, mostrou-se adequada. Além disso, demonstra ser uma importante ferramenta para direcionamento de projeto. Como continuidade dos estudos para validação dessa marcha de cálculo, sugere-se que se façam novos ensaios para verificar a ruptura dúctil, indicada pela região 2 de projeto, mais especificamente na transição entre as regiões 2 e 3.
A não utilização do mecanismo de incremento de ancoragem fez com que não se alcançasse o momento último, não registrando aumento de capacidade resistente da viga. O estudo sobre o início do descolamento da manta indicou que os métodos propostos pelo ACI e FIB estão adequados. Já o momento-fletor de início de descolamento proposto por Chaallal e equipe, apesar de diferir apenas $7 \%$ do valor encontrado no ensaio, mostrou-se contra a segurança.

\section{Referências bibliográficas}

AMERICAN CONCRETE INSTITUTE. State-of-the-art report on fiber reinforcing plastic reinforcement for concrete structures - ACI 440R-96. Reported by ACI Committee 440. Detroit, Michigan, 1996. 68p.

ARDUINI, M., TOMMASO, A. D., NANNI, A. Brittle failure in FRP plate and sheet bonded beams. ACI Structural Journal, v. 94, n. 4, p. 363-370, 1997.

ASHOUR, A. F., FAMILY, M. Tests of concrete flanged beams reinforced with CFRP bars. Magazine of Concrete Research, v. 58, n. 9, p. 627-639, 2006.

BARROS, J. A. O., DIAS, S. J. E. Near surface mounted CFRP laminates for shear strengthening of concrete beams. Cement \& Concrete Composites, n. 28, p. 276292, 2006.

CHAAllal, O., NOLLET, M. J. e PERRATON, D. Strengthening of reinforced concrete beams with externally bonded fiber-reinforced-plastic plates: design guidelines for shear and flexure. Canadian Journal of Civil Engineering, Montreal, v. 2, n. 25, p.692-704, 1998.

FÉDERATION INTERNATIONALE DU BÉTON. Externally bonded FRP reinforcement for RC structures-Technical Report-FIB Bulletin $n^{\circ} 14$. Lausanne, 2001. $130 \mathrm{p}$.

NG, S. C., LEE, S. A study of flexural behavior of reinforced concrete beam strengthened with carbon fiber-reinforced plastic (CFRP). Journal of Reinforced Plastics and Composites, v. 21, p. 919-938, 2002.

NORRIS, T., SAADATMANESH, H., EHSANI, M. R. Shear and flexural strengthening of R/C beams with carbon fiber sheets. ASCE Journal of Structural Engineering, v. 123, n.7 , p. 903-911, 1997.

PAPANICOLAOU, C. G. et al. Textile reinforced mortar (TRM) versus FRP as strengthening material of URM walls: out-of-plane cyclic loading. Materials and Structures, v. 41, p. 143-157, 2008.

ROBERTS, T. M. Approximate Analysis of Shear and Normal Stress Concentrations in the Adhesive Layer of Plated RC Beams. The Structural Engineer, v. 67, n. 12, p. 229-233, 1989.

SAADATMANESH, H., EHSANI, M. R. RC beams strengthened with GFRP plates. I: experimental study. ASCE Journal of Structural Engineering, v. 117, n. 11, p. 3417-3432, 1991.

SI-LARBI, A., FERRIER, E., HAMELIN, P. Flexural behaviour of MRBC beams (multi-reinforcing bars concrete beams), promoting the use of FRHPC. Composite Structures, v.74, p. 163-174, 2006.

SILVA, A. O. B. Reforço à flexão de vigas de concreto de elevada resistência por meio da colagem de manta polimérica reforçada com fibras de carbono. São Paulo: Faculdade de Engenharia Civil, Universidade Estadual de Campinas, 2001. 405p. (Dissertação de Mestrado).

Artigo recebido em 05 de outubro de 2011. Aprovado em 15 de abril de 2012. 